\title{
Interstellar $\mathrm{OH}^{+}, \mathrm{H}_{2} \mathrm{O}^{+}$and $\mathrm{H}_{3} \mathrm{O}^{+}$ along the sight-line to G10.6-0.4 ${ }^{\star}, \star \star$
}

\author{
M. Gerin ${ }^{1}$, M. De Luca ${ }^{1}$, J. Black ${ }^{2}$, J. R. Goicoechea ${ }^{3}$, E. Herbst ${ }^{4}$, D. A. Neufeld ${ }^{5}$, E. Falgarone ${ }^{1}$, B. Godard ${ }^{1,8}$, \\ J. C. Pearson ${ }^{6}$, D. C. Lis ${ }^{7}$, T. G. Phillips ${ }^{7}$, T. A. Bell ${ }^{7}$, P. Sonnentrucker ${ }^{5}$, F. Boulanger ${ }^{8}$, J. Cernicharo ${ }^{3}$, A. Coutens ${ }^{12,13}$, \\ E. Dartois 8 , P. Encrenaz ${ }^{1}$, T. Giesen ${ }^{9}$, P. F. Goldsmith ${ }^{6}$, H. Gupta $^{6}$, C. Gry ${ }^{10}$, P. Hennebelle ${ }^{1}$, P. Hily-Blant ${ }^{11}$, \\ C. Joblin ${ }^{12,13}$, M. Kazmierczak ${ }^{15}$, R. Kolos ${ }^{14}$, J. Krelowski ${ }^{15}$, J. Martin-Pintado ${ }^{3}$, R. Monje ${ }^{7}$, B. Mookerjea ${ }^{16}$, \\ M. Perault ${ }^{1}$, C. Persson ${ }^{2}$, R. Plume ${ }^{17}$, P. B. Rimmer ${ }^{4}$, M. Salez ${ }^{1}$, M. Schmidt ${ }^{18}$, J. Stutzki ${ }^{9}$, D. Teyssier ${ }^{19}$, \\ C. Vastel ${ }^{12,13}$, S. $\mathrm{Yu}^{6}$, A. Contursi ${ }^{20}$, K. Menten ${ }^{21}$, T. Geballe ${ }^{22}$, S. Schlemmer ${ }^{9}$, R. Shipman ${ }^{23}$, A. G. G. M. Tielens ${ }^{24}$, \\ S. Philipp-May ${ }^{21,25}$, A. Cros ${ }^{12,13}$, J. Zmuidzinas ${ }^{7}$, L. A. Samoska ${ }^{6}$, K. Klein ${ }^{25}$, and A. Lorenzani ${ }^{27}$
}

(Affiliations are available in the online edition)

Received 30 March 2010 / Accepted 30 April 2010

\section{ABSTRACT}

We report the detection of absorption lines by the reactive ions $\mathrm{OH}^{+}, \mathrm{H}_{2} \mathrm{O}^{+}$and $\mathrm{H}_{3} \mathrm{O}^{+}$along the line of sight to the submillimeter continuum source G10.6-0.4 (W31C). We used the Herschel HIFI instrument in dual beam switch mode to observe the ground state rotational transitions of $\mathrm{OH}^{+}$ at $971 \mathrm{GHz}, \mathrm{H}_{2} \mathrm{O}^{+}$at 1115 and $607 \mathrm{GHz}$, and $\mathrm{H}_{3} \mathrm{O}^{+}$at $984 \mathrm{GHz}$. The resultant spectra show deep absorption over a broad velocity range that originates in the interstellar matter along the line of sight to G10.6-0.4 as well as in the molecular gas directly associated with that source. The $\mathrm{OH}^{+}$spectrum reaches saturation over most velocities corresponding to the foreground gas, while the opacity of the $\mathrm{H}_{2} \mathrm{O}^{+}$lines remains lower than 1 in the same velocity range, and the $\mathrm{H}_{3} \mathrm{O}^{+}$line shows only weak absorption. For LSR velocities between 7 and $50 \mathrm{~km} \mathrm{~s}^{-1}$ we estimate total column densities of $N\left(\mathrm{OH}^{+}\right) \geq 2.5 \times 10^{14} \mathrm{~cm}^{-2}, N\left(\mathrm{H}_{2} \mathrm{O}^{+}\right) \sim 6 \times 10^{13} \mathrm{~cm}^{-2}$ and $N\left(\mathrm{H}_{3} \mathrm{O}^{+}\right) \sim 4.0 \times 10^{13} \mathrm{~cm}^{-2}$. These detections confirm the role of $\mathrm{O}^{+}$and $\mathrm{OH}^{+}$in initiating the oxygen chemistry in diffuse molecular gas and strengthen our understanding of the gas phase production of water. The high ratio of the $\mathrm{OH}^{+}$by the $\mathrm{H}_{2} \mathrm{O}^{+}$column density implies that these species predominantly trace low-density gas with a small fraction of hydrogen in molecular form.

Key words. ISM: general - ISM: molecules - ISM: clouds

\section{Introduction}

Oxygen chemistry in the interstellar medium is initiated by cosmic-ray or X-ray ionization of hydrogen. The resulting $\mathrm{H}^{+}$ and $\mathrm{H}_{3}^{+}$transfer their charge to form the $\mathrm{O}^{+}$and $\mathrm{OH}^{+}$ions, both of which react rapidly with $\mathrm{H}_{2}$ molecules. Subsequent $\mathrm{H}$-atom abstraction reactions proceed to the terminal ion $\mathrm{H}_{3} \mathrm{O}^{+}$with high efficiency, unless the electron fraction is unusually high. $\mathrm{H}_{3} \mathrm{O}^{+}$ does not react with $\mathrm{H}_{2}$ or any other abundant neutral species, but is destroyed by dissociative recombination with electrons. Recombination produces $\mathrm{O}, \mathrm{OH}$, and $\mathrm{H}_{2} \mathrm{O}$. This sequence is the main gas-phase chemical route to water in the interstellar medium. Until now, this classical scheme of the oxygen chemistry (Herbst \& Klemperer 1973) has been tested mainly through observations of $\mathrm{OH}$ and $\mathrm{H}_{2} \mathrm{O}$, whose abundances are affected by many other processes as well, and by a few observations of $\mathrm{H}_{3} \mathrm{O}^{+}$. With the HIFI instrument on the Herschel satellite it is now possible to observe the strongest transitions of light hydrides, including the reactive molecular ions $\mathrm{OH}^{+}$and $\mathrm{H}_{2} \mathrm{O}^{+}$that are thought to play crucial intermediate roles in the formation of $\mathrm{OH}$ and $\mathrm{H}_{2} \mathrm{O}$.

Previous attempts to observe interstellar $\mathrm{H}_{2} \mathrm{O}^{+}$and $\mathrm{OH}^{+}$ from optical spectroscopy are very limited. Smith et al. (1984)

^ Herschel is an ESA space observatory with science instruments provided by European-led Principal Investigator consortia and with important particiation from NASA.

$\star \star$ Figure 2 is only available in electronic form at http://www . aanda.org report an upper limit of $10^{12} \mathrm{~cm}^{-2}$ for $\mathrm{H}_{2} \mathrm{O}^{+}$based on optical absorption spectroscopy. No observation of the $\mathrm{OH}^{+}$transition at $358.4 \mathrm{~nm}$ has been reported until very recently (Krelowski et al., private communication). Submillimeter telescopes have been more successful in detecting these ions. With the Atacama Pathfinder EXperiment (APEX) telescope Wyrowski et al. (2010) detected two hyperfine-structure components of the $\mathrm{OH}^{+}$ $N=1-0 \mathrm{~J}=0-1$ transition near $909.2 \mathrm{GHz}$ towards the strong continuum source Sgr B2(M) and concluded that $\mathrm{OH}^{+}$plays a major role in the chemistry of the diffuse molecular gas. Using HIFI on Herschel, Ossenkopf et al. (2010) report the detection of $\mathrm{O}-\mathrm{H}_{2} \mathrm{O}^{+}$absorption in the three massive star-forming regions NGC 6334, Sgr B2, and DR 21.

We present observations toward the massive star-forming region G10.6-0.4 (W 31C) located at $R A=18^{\mathrm{h}} 10^{\mathrm{m}} 28.70^{\mathrm{s}}$, Dec $=$ $-19^{\circ} 55^{\prime} 50 \mathrm{~s}$ (J2000). The submillimeter-wave continuum emission is associated with a cluster of ultra-compact $\mathrm{H}$ II regions located in the so-called " $30 \mathrm{~km} \mathrm{~s}^{-1}$ arm" at a kinematic distance of $4.8 \mathrm{kpc}$ (Fish et al. 2003; Corbel \& Eikenberry 2004). The molecular cloud surrounding the star-forming complex shows evidence of continuing accretion onto the embedded stars (Keto \& Wood 2006; Sollins \& Ho 2005). As discussed by Neufeld et al. (2010), line absorption by foreground gas has been detected from [OI] (Keene et al. 1999), ${ }^{13} \mathrm{CH}^{+}$(Falgarone et al. 2005), $\mathrm{HCO}^{+}, \mathrm{HCN}, \mathrm{HNC}, \mathrm{CN}$ (Godard et al. 2010), and atomic hydrogen (Fish et al. 2003). While the gas associated directly with G10.6-0.4 is detected in the velocity range -5 to $+5 \mathrm{~km} \mathrm{~s}^{-1}$, the 
Table 1. Transition spectroscopic parameters.

\begin{tabular}{lccccc}
\hline \hline Transition & $\begin{array}{c}\text { Frequency } \\
\mathrm{MHz}\end{array}$ & $\begin{array}{c}\text { Error } \\
\mathrm{MHz}\end{array}$ & $\begin{array}{c}E_{l} \\
\mathrm{~cm}^{-1}\end{array}$ & $\begin{array}{c}A \\
10^{-2} \mathrm{~s}^{-1}\end{array}$ & Ref. \\
\hline $\mathrm{OH}^{+} N=1-0$ & & & & & \\
$2,5 / 2-1,3 / 2$ & 971803.8 & 1.5 & 0.0 & 1.82 & 1 \\
$2,3 / 2-1,1 / 2$ & 971805.3 & 1.5 & 0.0 & 1.52 & 1 \\
$2,3 / 2-1,3 / 2$ & 971919.2 & 1.0 & 0.0 & 0.30 & 1 \\
\hline $\mathrm{o}-\mathrm{H}_{2} \mathrm{O}^{+} 1_{1,1}-0_{0,0}$ & & & & & \\
$3 / 2,3 / 2-1 / 2,1 / 2$ & 1115122.0 & 10 & 0.0 & 1.71 & 2 \\
$3 / 2,1 / 2-1 / 2,1 / 2$ & 1115158.0 & 10 & 0.0 & 2.75 & 2 \\
$3 / 2,5 / 2-1 / 2,3 / 2$ & 1115175.8 & 10 & 0.0 & 3.10 & 2 \\
$3 / 2,3 / 2-1 / 2,3 / 2$ & 1115235.6 & 10 & 0.0 & 1.39 & 2 \\
$3 / 2,1 / 2-1 / 2,3 / 2$ & 1115271.6 & 10 & 0.0 & 0.35 & 2 \\
\hline $\mathrm{p}^{-} \mathrm{H}_{2} \mathrm{O}^{+} 1_{1,0}-1_{0,1}$ & & & & & \\
$3 / 2,3 / 2-3 / 2,3 / 2$ & 607207.0 & 20 & 20.9 & 0.60 & 2 \\
$\mathrm{H}_{3} \mathrm{O}^{+}$ & & & & & \\
$0_{0}^{-}-1_{0}^{+}$ & 984711.9 & 0.3 & 5.1 & 2.3 & 3 \\
\hline
\end{tabular}

References. 1, Müller et al. (2005) \& CDMS; 2, Strahan et al. (1986), Ossenkopf et al. (2010); 3 Yu et al. (2009) \& JPL catalog.

foreground gas along the line of sight is confined to the velocity interval from $\sim 5$ to $45 \mathrm{~km} \mathrm{~s}^{-1}$.

\section{Spectroscopy}

Table 1 summarizes the spectroscopic parameters used for the searched transitions. The $\mathrm{OH}^{+}$ion has a ${ }^{3} \Sigma^{-}$electronic ground state. We used the data from Beekoy et al. (1985) with reported uncertainties for the line frequencies better than about 1.5 MHz. Müller et al. (2005) have published a global fit using these data which is available in the Cologne Data base for Molecular Spectroscopy (CDMS). The ground state dipole moment obtained from theoretical calculations is $2.3 \mathrm{D}$ (Werner et al. 1983; Cheng et al. 2007).

The spectroscopic properties of $\mathrm{H}_{2} \mathrm{O}^{+}$are extensively discussed in the accompanying letter (Ossenkopf et al. 2010). Its dipole moment is very similar to that of $\mathrm{OH}^{+}$, as recently calculated by $\mathrm{Wu}$ et al. (2004), who found the ground state dipole moment to be 0.933 , mistakenly printed in atomic units for a value of 2.370 Debye. This molecular ion has ortho and para symmetry states, which have the opposite parity to the water vapor molecule, i.e. the lowest state is the ortho state. Therefore the ortho/para ratio should be at least 3:1.

$\mathrm{H}_{3} \mathrm{O}^{+}$has been the subject of many spectroscopic investigations, because for the frequency of the inversion transition of this molecular ion is comparable to the rotational constant. The most recent analysis has been presented by Yu et al. (2009) and is available in the JPL spectral line catalog. We observed the lowest transition from the E symmetry state, which is $\sim 5 \mathrm{~cm}^{-1}$ above the ground state.

\section{Observations}

The observations were carried out by the HIFI instrument on board Herschel (Pilbratt et al. 2010; de Graauw et al. 2010), in the double beam switching (DBS) mode. We used the wide band spectrometer (WBS) with $4 \mathrm{GHz}$ bandwidth and $1.1 \mathrm{MHz}$ effective spectral resolution. To help separate the signals from the lower and upper sidebands, three integrations were carried out at each frequency, with the local oscillator (LO) shifted by $15 \mathrm{~km} \mathrm{~s}^{-1}$.

The data were first processed with HIPE Ott et al. (2010) and subsequently exported to CLASS. We checked that the target lines did not suffer from any contamination by lines from the other sideband and that the absorption features were not contaminated by emission in the reference beam. The two polarizations excellently agree - the continuum levels agree to better than $10 \%$ - except for the $\mathrm{OH}^{+}$line, where the $\mathrm{V}$ polarization shows ripples and has therefore not been used in the present analysis. We combined the data from the three LO tunings and the two polarizations to obtain the average spectra shown in Fig. 1. For double-side band mixers with equal sideband gains, fully saturated lines are expected to absorb only half of the continuum signal. This expectation is borne out in the present data. While the $\mathrm{OH}^{+}$line is clearly saturated, both $\mathrm{H}_{2} \mathrm{O}^{+}$lines show moderate absorption, while the $\mathrm{H}_{3} \mathrm{O}^{+}$line only shows weak absorption.

\section{Results}

To understand the structure of the absorption profiles and derive column densities of the reactive ions, we normalized the spectra by the SSB continuum intensities. We constructed empirical models of the line profiles by combining multiple velocity components, defined by their central velocity, line width, and opacity. The predicted spectrum was then computed by combining the contributions of the hyperfine components $\left(3\right.$ for $\mathrm{OH}^{+}$and 5 for $\mathrm{H}_{2} \mathrm{O}^{+}$as listed in Table 1) to the overall line profile. We show in Fig. 1 spectra normalized by the continuum intensities, with the synthetic spectra superimposed. Table 2 lists the model parameters.

Although we obtained good fits of the $\mathrm{OH}^{+}$and $\mathrm{H}_{2} \mathrm{O}^{+}$profiles, it is likely that the solution is not unique because the number of velocity components is not well constrained. The $\mathrm{OH}^{+}$ ground state lines are known with a good accuracy of $\sim 1.5 \mathrm{MHz}$. Velocities obtained by the empirical model agree well with the velocities where deep $\mathrm{HCO}^{+}(1-0)$ (Keene et al. 1999; Godard et al. 2010), p- $\mathrm{H}_{2} \mathrm{O}$ and $\mathrm{HF}$ (Neufeld et al. 2010) and HI Fish et al. (2003) absorptions are detected. It is remarkable that one of the strongest $\mathrm{OH}^{+}$absorption features at $V_{\mathrm{LSR}} \sim 7 \mathrm{~km} \mathrm{~s}^{-1}$ coincides with a strong dip in the HI profile that has no counterpart in $\mathrm{CO}$ emission Falgarone et al. (2005). Overall, the velocities of the $\mathrm{OH}^{+}$and $\mathrm{H}_{2} \mathrm{O}^{+}$velocity components agree well. Although the lines are detected in absorption, given the similarity of their Local Standard of Rest (LSR) velocities with the rest velocity of G10.6-0.4, it is likely that the most blueshifted velocity components are associated with the G10.6-0.4 molecular complex.

Below we discuss the column densities and abundances of $\mathrm{OH}^{+}, \mathrm{H}_{2} \mathrm{O}^{+}$, and $\mathrm{H}_{3} \mathrm{O}^{+}$in the foreground interstellar gas, with LSR velocities higher than $5 \mathrm{~km} \mathrm{~s}^{-1}$. Neufeld et al. (2010) discuss the total column density of this material and obtain a column density of hydrogen in all forms of $N_{\mathrm{H}} \sim 2.7 \times 10^{22} \mathrm{~cm}^{-2}$. This figure is consistent with the lower limit derived from the oxygen column density measured with ISO, $5 \times 10^{18} \mathrm{~cm}^{-2}$ (Keene et al. 1999), which gives $N_{\mathrm{H}}>1.7 \times 10^{22} \mathrm{~cm}^{-2}$ using $\mathrm{O} / \mathrm{H}=3 \times 10^{-4}$. For this line of sight the estimated atomic hydrogen column density is at most $N(\mathrm{HI})=1.2 \times 10^{22} \mathrm{~cm}^{-2}$ (Godard et al. 2010), hence the average fraction of hydrogen in molecular form is $f\left(\mathrm{H}_{2}\right) \sim 0.6$, with significant variations among the velocity components.

The total $\mathrm{OH}^{+}$and $\mathrm{H}_{2} \mathrm{O}^{+}$column densities were obtained by summing the contributions of the velocity components with centroids larger than $7 \mathrm{kms}^{-1}$. We derive $N\left(\mathrm{OH}^{+}\right) \geq 2.5 \times 10^{14} \mathrm{~cm}^{-2}$ and $N\left(\mathrm{o}-\mathrm{H}_{2} \mathrm{O}^{+}\right)=4.4 \times 10^{13} \mathrm{~cm}^{-2}$. Although the absorption from the $\mathrm{p}-\mathrm{H}_{2} \mathrm{O}^{+}$line at $607 \mathrm{GHz}$ is blended with emission from $\mathrm{H}^{13} \mathrm{CO}^{+}(6-5)$ at $607175 \mathrm{MHz}$, weak absorption is clearly detected in the velocity range of the foreground gas along the line of sight to G10.6-0.4. The integrated opacity for the LSR velocity range 20 to $45 \mathrm{~km} \mathrm{~s}^{-1}$ is $\int \tau \mathrm{d} v=2 \mathrm{~km} \mathrm{~s}^{-1}$, corresponding to a total column density of $\sim 1 \times 10^{13} \mathrm{~cm}^{-2}$. This figure is obtained for a limited velocity range, and should therefore be considered a lower limit. Given the uncertainties, the data 

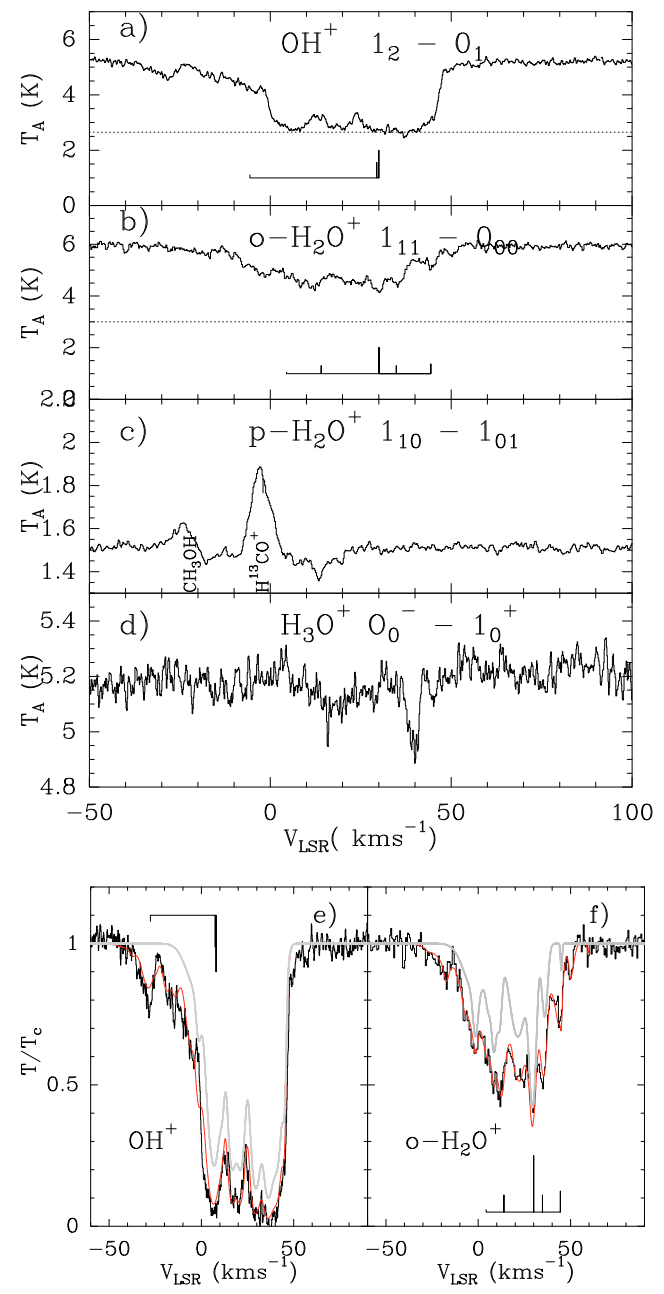

Fig. 1. HIFI spectra of a) $\mathrm{OH}^{+}$; b) o- $\mathrm{H}_{2} \mathrm{O}^{+}$; c) $p-\mathrm{H}_{2} \mathrm{O}^{+}$and (d) $\mathrm{H}_{3} \mathrm{O}^{+}$. The hyperfine structure of the $\mathrm{OH}^{+}$and $\mathrm{o}-\mathrm{H}_{2} \mathrm{O}^{+}$lines (see Table 1) is indicated. In both cases, the velocity scale corresponds to the rest frequency of the strongest hyperfine component. In panel c), the velocity scale refers to the frequency of the $\mathrm{H}^{13} \mathrm{CO}^{+}$(6-5) line at 607174.65 MHz. Panel d) uses the frequency of $984711.907 \mathrm{MHz}$. Panels e) and f) show overlays with the synthetic spectra constructed with the empirical models detailed in Table 2. Grey curves show the profile of the strongest hyperfine component.

are consistent with an $\mathrm{o} / \mathrm{p}$ ratio of 3 for $\mathrm{H}_{2} \mathrm{O}^{+}$, as expected from the spin statistics. The total column density of $\mathrm{H}_{2} \mathrm{O}^{+}$is therefore $N\left(\mathrm{H}_{2} \mathrm{O}^{+}\right) \sim 6 \times 10^{13} \mathrm{~cm}^{-2}$ and the abundance ratio $N\left(\mathrm{OH}^{+}\right) / N\left(\mathrm{H}_{2} \mathrm{O}^{+}\right) \geq 4$. Using the total gas column density, we conclude that $\mathrm{OH}^{+}$and $\mathrm{H}_{2} \mathrm{O}^{+}$reach fractional abundances, of $N\left(\mathrm{OH}^{+}\right) / N_{\mathrm{H}} \geq \times 10^{-8}$ and $N\left(\mathrm{H}_{2} \mathrm{O}^{+}\right) / N_{\mathrm{H}} \sim 2 \times 10^{-9}$.

The $\mathrm{H}_{3} \mathrm{O}^{+}$spectrum shows a sharp feature with strongest absorption at $V_{\mathrm{LSR}} \sim 40 \mathrm{~km} \mathrm{~s}^{-1}$ and a broad component centered at $V_{\mathrm{LSR}} \sim 20 \mathrm{~km} \mathrm{~s}^{-1}$. This velocity structure is more similar to the $\mathrm{HF}$ or $\mathrm{p}-\mathrm{H}_{2} \mathrm{O}$ spectra than the $\mathrm{OH}^{+}$and $\mathrm{H}_{2} \mathrm{O}^{+}$velocity structure, confirming that these molecular ions trace different physical conditions. We derived the $\mathrm{H}_{3} \mathrm{O}^{+}$column density of the foreground gas for all velocity components. Assuming an excitation temperature of $5 \mathrm{~K}$, appropriate for the conditions of the gas in the inner galaxy, we derive $N\left(\mathrm{H}_{3} \mathrm{O}^{+}\right) \sim 4 \times 10^{13} \mathrm{~cm}^{-2}$ for the LSR velocity range $7-45 \mathrm{~km} \mathrm{~s}^{-1}$. Therefore $\mathrm{N}\left(\mathrm{OH}^{+}\right) / \mathrm{N}^{-}\left(\mathrm{H}_{3} \mathrm{O}^{+}\right)$ $>6$ and $\mathrm{N}\left(\mathrm{H}_{2} \mathrm{O}^{+}\right) / \mathrm{N}\left(\mathrm{H}_{3} \mathrm{O}^{+}\right) \sim 1.5$. These numbers represent the average for all velocity components but, given the different velocity structure of $\mathrm{H}_{3} \mathrm{O}^{+}$and $\mathrm{OH}^{+}$, the column density ratio for individual velocity components can significantly differ from the average value.
Table 2. Empirical models used to analyze the $\mathrm{OH}^{+}$and $\mathrm{H}_{2} \mathrm{O}^{+}$spectra.

\begin{tabular}{l|rll|rll}
\hline \hline & \multicolumn{3}{|c|}{$\mathrm{OH}^{+}$} & \multicolumn{3}{c}{$\mathrm{H}_{2} \mathrm{O}^{+}$} \\
$V_{\mathrm{LSR}}$ & $\Delta_{V}$ & $\tau$ & $\mathrm{N}$ & $\Delta_{V}$ & $\tau$ & $N$ \\
$\mathrm{~km} \mathrm{~s}^{-1}$ & $\mathrm{~km} \mathrm{~s}^{-1}$ & & $10^{13} \mathrm{~cm}^{-2}$ & $\mathrm{~km} \mathrm{~s}^{-1}$ & & $10^{12} \mathrm{~cm}^{-2}$ \\
\hline-1.0 & 14.0 & 0.2 & 1.3 & 14.0 & 0.2 & 12 \\
-1.5 & 3.0 & 0.2 & 0.3 & 3.0 & 0.2 & 2.5 \\
5.5 & 6.0 & 1.0 & 2.8 & 3.0 & 0.15 & 1.9 \\
8.5 & 5.0 & 0.8 & 1.9 & 3 & 0.4 & 5.0 \\
11.5 & 3.0 & 0.5 & 0.7 & 3.0 & 0.3 & 3.8 \\
16.5 & 5.0 & 1.5 & 3.5 & & & \\
21.5 & 5.0 & 1.5 & 3.5 & 10.0 & 0.4 & 1.7 \\
29.5 & 6.0 & 2.0 & 5.6 & 4.0 & 0.8 & 1.3 \\
36.0 & 5.0 & 2.0 & 4.7 & 3.5 & 0.3 & 4.4 \\
41.0 & 6.0 & 1.5 & 4.2 & & & \\
45.0 & 2.0 & 0.5 & 0.5 & 1.0 & 0.1 & 0.4 \\
\hline
\end{tabular}

Notes. The $\mathrm{OH}^{+}$and $\mathrm{H}_{2} \mathrm{O}^{+}$parameters refer to the frequency of the strongest hyperfine component (cf. Table 1).

\section{Chemistry}

\subsection{PDR models}

In order to model the $\mathrm{OH}^{+}$and $\mathrm{H}_{2} \mathrm{O}^{+}$chemistry in diffuse clouds, we used the Meudon PDR code ${ }^{1}$, a photochemical model of a plane parallel and stationary PDR (Le Petit et al. 2006; Goicoechea \& Le Bourlot 2007). We present models for a representative cloud of $A_{V}=1 \mathrm{mag}$ illuminated from both sides. Although the total extinction along this line of sight is $\sim 14 \mathrm{mag}$ (Corbel \& Eikenberry 2004; Neufeld et al. 2010), the line profile clearly shows multiple velocity components, revealing interstellar clouds along the line of sight. As discussed by Godard et al. (2010), each of these clouds has low to moderate extinctions, corresponding to diffuse or transluscent matter.

The Meudon PDR code computes the gas temperature gradient by solving the thermal balance (see Le Petit et al. 2006). Our pure gas-phase chemical network is based on a modified version of the Ohio State University (OSU) gas-phase network updated for photochemical studies. The extinction of UV and visible radiation is computed using a dust grain size distribution following a power-law (Mathis et al. 1977). The adopted oxygen and carbon elemental abundances are $3.02 \times 10^{-4}$ and $1.38 \times 10^{-4}$, respectively. As a starting point we used an ionization rate (of $\mathrm{H})$ due to cosmic rays of $\zeta_{\mathrm{H}}=2.3 \times 10^{-17} \mathrm{~s}^{-1}$.

The oxygen chemistry starts with the $\left.\mathrm{O}^{3} \mathrm{P}_{J}\right)+\mathrm{H}^{+} \rightarrow \mathrm{O}^{+}\left({ }^{4} \mathrm{~S}\right)$ $+\mathrm{H}$ charge transfer process, where reaction rates are sensitive to the oxygen fine-structure level populations (Stancil et al. 1999, and references therein). Indeed the charge transfer proceeds quickly when the $\mathrm{O}\left({ }^{3} \mathrm{P}_{1}\right)$ level $\left(\Delta E_{12} / k=228 \mathrm{~K}\right)$ is significantly populated, but it can be a slow proceess in cold gas where only the $\mathrm{O}\left({ }^{3} \mathrm{P}_{2}\right)$ ground-state level is populated (Spirko et al. 2003). As listed in Table $3, \mathrm{OH}^{+}$is formed by $\mathrm{O}^{+}+\mathrm{H}_{2}$ with additional contribution from $\mathrm{OH}$ photoionization at the cloud edges, and by $\mathrm{O}+\mathrm{H}_{3}^{+}$. Therefore, the $\mathrm{OH}^{+}$abundance may be sensitive to gas temperature, far ultraviolet (FUV) radiation field and $\zeta_{\mathrm{H}}$.

In order to investigate the possible sources of the high $\mathrm{OH}^{+} / \mathrm{H}_{2} \mathrm{O}^{+}$abundance ratio, Fig. 2 shows photochemical models for different densities, and radiation fields (thus different gas temperatures). High $\mathrm{OH}^{+} / \mathrm{H}_{2} \mathrm{O}^{+}$abundance ratios are found in the warm gas at low extinction, where the fraction of hydrogen in molecular form is significantly smaller than 1 . As a limited set reactions need to be considered we built a simplified analytical model based on the network listed in Table 3, and find

$n\left(\mathrm{OH}^{+}\right) / n\left(\mathrm{H}_{2} \mathrm{O}^{+}\right)=\left(k_{2} / k_{1}\right)+\left(k_{3} / k_{1}\right)\left[n\left(\mathrm{e}^{-}\right) / n\left(\mathrm{H}_{2}\right)\right]$

\footnotetext{
${ }^{1}$ See http://pdr.obspm.fr/PDRcode.html
} 
Table 3. Main chemical reactions.

\begin{tabular}{llc}
\hline \hline Number & Reaction & Rate $\left(\mathrm{cm}^{-3} \mathrm{~s}^{-1}\right)$ \\
\hline 1 & $\mathrm{OH}^{+}+\mathrm{H}_{2} \rightarrow \mathrm{H}_{2} \mathrm{O}^{+}+\mathrm{H}$ & $k_{1}=1.01 \times 10^{-9}$ \\
2 & $\mathrm{H}_{2} \mathrm{O}^{+}+\mathrm{H}_{2} \rightarrow \mathrm{H}_{3} \mathrm{O}^{+}+\mathrm{H}$ & $k_{2}=6.4 \times 10^{-10}$ \\
3 & $\mathrm{H}_{2} \mathrm{O}^{+}+\mathrm{e}^{-} \rightarrow$ products & $k_{3}=4.3 \times 10^{-7}(T / 300 \mathrm{~K})^{-0.5}$ \\
4 & $\mathrm{H}_{3} \mathrm{O}^{+}+\mathrm{e}^{-} \rightarrow$ products & $k_{4}=4.3 \times 10^{-7}(T / 300 \mathrm{~K})^{-0.5}$ \\
5 & $\mathrm{OH}^{+}+\mathrm{e}^{-} \rightarrow$ products & $k_{5}=3.75 \times 10^{-8}(T / 300 \mathrm{~K})^{-0.5}$ \\
\hline
\end{tabular}

$=0.64+430 \times(T / 300)^{-0.5} \times\left[n\left(\mathrm{e}^{-}\right) / n\left(\mathrm{H}_{2}\right)\right]$.

Using $\left.f\left(\mathrm{H}_{2}\right)=2 n\left(\mathrm{H}_{2}\right) /\left(n(\mathrm{H})+2 n\left(\mathrm{H}_{2}\right)\right)=2 n\left(\mathrm{H}_{2}\right) / n_{\mathrm{H}}\right)$ and assuming that all carbon is ionised $\left(n\left(\mathrm{e}^{-}\right)=n_{C}=1.4 \times\right.$ $\left.10^{-4} n_{\mathrm{H}}\right)$ Eq. (2) leads to $n\left(\mathrm{OH}^{+}\right) / n\left(\mathrm{H}_{2} \mathrm{O}^{+}\right)=0.64+0.12 \times$ $(T / 300)^{-0.5} / f\left(\mathrm{H}_{2}\right)$. The data suggest that $N\left(\mathrm{OH}^{+}\right) / N\left(\mathrm{H}_{2} \mathrm{O}^{+}\right)>$ 4 , implying that $f\left(\mathrm{H}_{2}\right)<0.06$ at $T=100 \mathrm{~K}$, an appropriate temperature for diffuse gas in the molecular ring. Equation (2) determined for each Av in the model is shown as green lines in Fig. 2. It fits the modeled abundance perfectly, demonstrating that the main ingredients have been captured by this simple model. Note that the reactions were studied in the laboratory and their rates are known with a good accuracy.

\subsection{The role of cosmic rays}

Like $\mathrm{H}_{3}^{+}$(Indriolo et al. 2007), $\mathrm{OH}^{+}$and $\mathrm{H}_{2} \mathrm{O}^{+}$can be used to trace the cosmic ray ionization rate for $\mathrm{H}$ atoms $\zeta_{\mathrm{H}}$. In the moderately warm gas where they are produced, the charge exchange reaction is rapid. Then, every cosmic ray ionization of $\mathrm{H}$ can lead to $\mathrm{O}^{+}$and we can use the measured $\mathrm{OH}^{+}$abundance to set limits on $\zeta_{\mathrm{H}}$. In addition to the reaction with $\mathrm{H}_{2}, \mathrm{OH}^{+}$is destroyed in dissociative recombination reactions with electrons. This implies $\zeta_{\mathrm{H}}>X\left(\mathrm{OH}^{+}\right) x\left(\mathrm{e}^{-}\right) n(\mathrm{H}) k_{5}, \zeta_{\mathrm{H}}>9.2 \times 10^{-12} \times n\left(\mathrm{OH}^{+}\right)$at $T=100 \mathrm{~K}$.

The lower limit on the $\mathrm{OH}^{+}$abundance in the atomic gas is $n\left(\mathrm{OH}^{+}\right) / n(\mathrm{H})>2 \times 10^{-8}$, which places a robust lower limit of $\zeta_{\mathrm{H}}>1.8 \times 10^{-19} n(\mathrm{H})$. This lower limit is very conservative: depending on the conditions in the absorbing material, higher values of $\zeta_{\mathrm{H}}$ may prove necessary to account for the observed column densities of $\mathrm{OH}^{+}$and $\mathrm{H}_{2} \mathrm{O}^{+}$. The $\mathrm{H}_{3}^{+}$observations of diffuse interstellar clouds point toward an enhanced ionization rate at low extinction. In a future analysis we will consider how $\mathrm{OH}^{+}$ and $\mathrm{H}_{2} \mathrm{O}^{+}$can provide additional constraints upon $\zeta_{\mathrm{H}}$.

\subsection{The role of turbulence}

In the warm chemistry driven by intermittent turbulent dissipation (Godard et al. 2009), the production of $\mathrm{OH}^{+}$and $\mathrm{H}_{2} \mathrm{O}^{+}$ is enhanced compared to UV-driven chemistry, with an efficiency depending only on the turbulent dissipation rate, $\epsilon$. For $\epsilon=2 \times 10^{-24} \mathrm{ergcm}^{-3} \mathrm{~s}^{-1}$, ten times higher than the average Galactic value appropriate for the inner Galaxy, the TDR model predicts $N\left(\mathrm{CH}^{+}\right)=4.3 \times 10^{13} \mathrm{~cm}^{-3}, N\left(\mathrm{OH}^{+}\right)=2 \times 10^{12} \mathrm{~cm}^{-2}$ and $N\left(\mathrm{H}_{2} \mathrm{O}^{+}\right)=2.2 \times 10^{12} \mathrm{~cm}^{-2}$ per magnitude of gas of density $n_{\mathrm{H}}=50 \mathrm{~cm}^{-3}$. The ratios between these three species weakly depend on $\epsilon$. With the observed $\mathrm{CH}^{+}$column density, $6.7 \times 10^{14} \mathrm{~cm}^{-2}$, derived from ${ }^{13} \mathrm{CH}^{+}\left(\right.$Falgarone et al. 2005) ${ }^{2}$, we therefore expect the following contributions of the TDR chemistry in that line of sight: $N\left(\mathrm{OH}^{+}\right)=3.1 \times 10^{13} \mathrm{~cm}^{-2}$, and $N\left(\mathrm{H}_{2} \mathrm{O}^{+}\right)=3.4 \times 10^{13} \mathrm{~cm}^{-2}$. The $\mathrm{OH}^{+}$column is well below the observed value, while that of $\mathrm{H}_{2} \mathrm{O}^{+}$is interestingly close.

\section{Conclusion}

The detection of interstellar $\mathrm{OH}^{+}, \mathrm{H}_{2} \mathrm{O}^{+}$, and $\mathrm{H}_{3} \mathrm{O}^{+}$provides a strong support to our understanding of the gas phase oxygen chemistry, in particular the gas phase route to water vapor. The combined analysis of the $\mathrm{OH}^{+}$and $\mathrm{H}_{2} \mathrm{O}^{+}$ground state spectra has shown that they mostly reside in warmish atomic gas where they are produced in a reaction sequence initiated by atomic $\mathrm{H}$ ionization followed by rapid reaction with traces of $\mathrm{H}_{2}$. Their abundances and abundance ratio can be used to constrain the fraction of hydrogen in molecular form, and to set a lower limit for the cosmic ray ionization in the diffuse gas, where few other diagnostics are applicable. As these reactive molecular ions can be observed from space with the Herschel Space Observatory, and in exceptional conditions from the ground, these preliminary conclusions should be verified by more extensive observations. It is also expected that strong features from both $\mathrm{OH}^{+}$and $\mathrm{H}_{2} \mathrm{O}^{+}$ will be detected in the spectra of distant galaxies, where elevated fluxes of ionizing radiation are produced by active nuclei and intense star-formation activity.

Acknowledgements. HIFI has been designed and built by a consortium of institutes and university departments from across Europe, Canada and the United States under the leadership of SRON, Netherlands Institute for Space Research, Groningen, The Netherlands, and with major contributions from Germany, France and the US. Consortium members are: Canada: CSA, U. Waterloo; France: CESR, LAB, LERMA, IRAM; Germany: KOSMA, MPIfR, MPS; Ireland: NUI Maynooth; Italy: ASI, IFSI-INAF, Osservatorio Astrofisico di Arcetri-INAF; Netherlands: SRON, TUD; Poland: CAMK, CBK; Spain: Observatorio Astronòmico Nacional (IGN), Centro de Astrobiologia; Sweden: Chalmers University of Technology - MC2, RSS \& GARD, Onsala Space Observatory, Swedish National Space Board, Stockholm University - Stockholm Observatory; Switzerland: ETH Zurich, FHNW; USA: CalTech, JPL, NHSC. MG, EF, MDL acknowledge the support from CNES, and ANR through the SCHISM project (ANR-09-BLAN-231). M.S. acknowledges support from grant N203393334 (MNiSW).

\section{References}

Beekoy, J. P., Verhoeve, P., Meerts, W. L., et al. 1985, J. Chem. Phys., 82, 3868 Cheng, M., Brown, J. M., Rosmus, P., et al. 2007, Phys. Rev. A, 75, 012502 Corbel, S., \& Eikenberry, S. S. 2004, A\&A, 419, 191 Falgarone, E., Phillips, T. G., Pearson, J. C. 2005, ApJ, 634, L149 Fish, V. L., Reid, M. J., Wilner, D. J., et al. 2003, ApJ, 587, 701 Godard, B., Falgarone, E., Pineau, des Forêts, G. 2009, A\&A, 495, 847 Godard, B., Falgarone, E., Gerin, M., et al. 2010, A\&A, accepted Goicoechea, J. R., \& Le Bourlot, J. 2007, A\&A, 467, 1 de Graauw, T., Helmich, F. P., Phillips, T. G., et al. 2010, A\&A, 518, L6 Herbst, E., \& Klemperer, W. 1973, ApJ, 185, 505

Indriolo, N., Geballe, T. R., Oka, T., \& McCall, B. J. 2007, ApJ, 671, 1736 Keene, J., Lis, D. C., Phillips, T. G., et al. 1999, ESASP, 427, 687 Keto, E., \& Wood, K. 2006, ApJ, 637, 85

Le Petit, F., Nehmé, C., Le Bourlot, J., \& Roueff, E. 2006, ApJS, 164, 506 Mathis, J. S., Rumpl, W., \& Nordsieck, K. H. 1977, ApJ, 217, 425

Müller, H. S. P., Schlöder, F., Stutzki, J., et al. 2005, Mol. Struct., 742, 215 Neufeld, D. A., Sonnentrucker, P., Phillips, T. G., et al. 2010, A\&A, 518, L108 Ossenkopf, V., Müller, H. S. P., Lis, D. C., et al. 2010, A\&A, 518, L111 Ott S., 2010, in Astronomical Data analysis Software and Systems XIX, ed. Y. Mizumoto, K. I. Morita, \& M. Ohishi, ASP Conf. Ser., in press

Pilbratt, G., Riedinger, J. L., Passvogel, T., et al. 2010, A\&A, 518, L1 Smith, W. H., Schempp, W. V., \& Federman, S. R. 1984, ApJ, 277, 196 Sollins, P. K., \& Ho, P. T. P. 2005, ApJ, 630, 987

Spirko, J. A., Zirbel, J. J., \& Hickman, A. P. 2003, JPhB, 36, 1645 Stancil, P. C., Schultz, D. R., Kimura, M., et al. 1999, A\&AS, 140, 225 Strahan, S. E., Müller, R. P., \& Saykally, R. J. 1986, J. Chem. Phys., 85, 1252 Werner, H.-J., Rosmus, P., \& Reinsch, E.-A. 1983, J. Chem. Phys., 79, 905 Wyrowski, F., Menten, K. M., Güsten, R., \& Belloche, A. 2010, A\&A, accepted [arkiv: 1004.2627]

Wu, S., Chen, Y., Yang, X., et al. 2004, J. Mol. Spectrosc., 225, 96 Yu, S., Drouin, B. J., Pearson, J. C., \& Pickett, H. M. 2009, ApJS, 180, 119

\footnotetext{
${ }^{2}$ In that paper it was underestimated by a factor 8 because of an unjustified approximation made in the derivation of the opacity.
} 
M. Gerin et al.: Interstellar $\mathrm{OH}^{+}, \mathrm{H}_{2} \mathrm{O}^{+}$and $\mathrm{H}_{3} \mathrm{O}^{+}$along the sight-line to G10.6-0.4
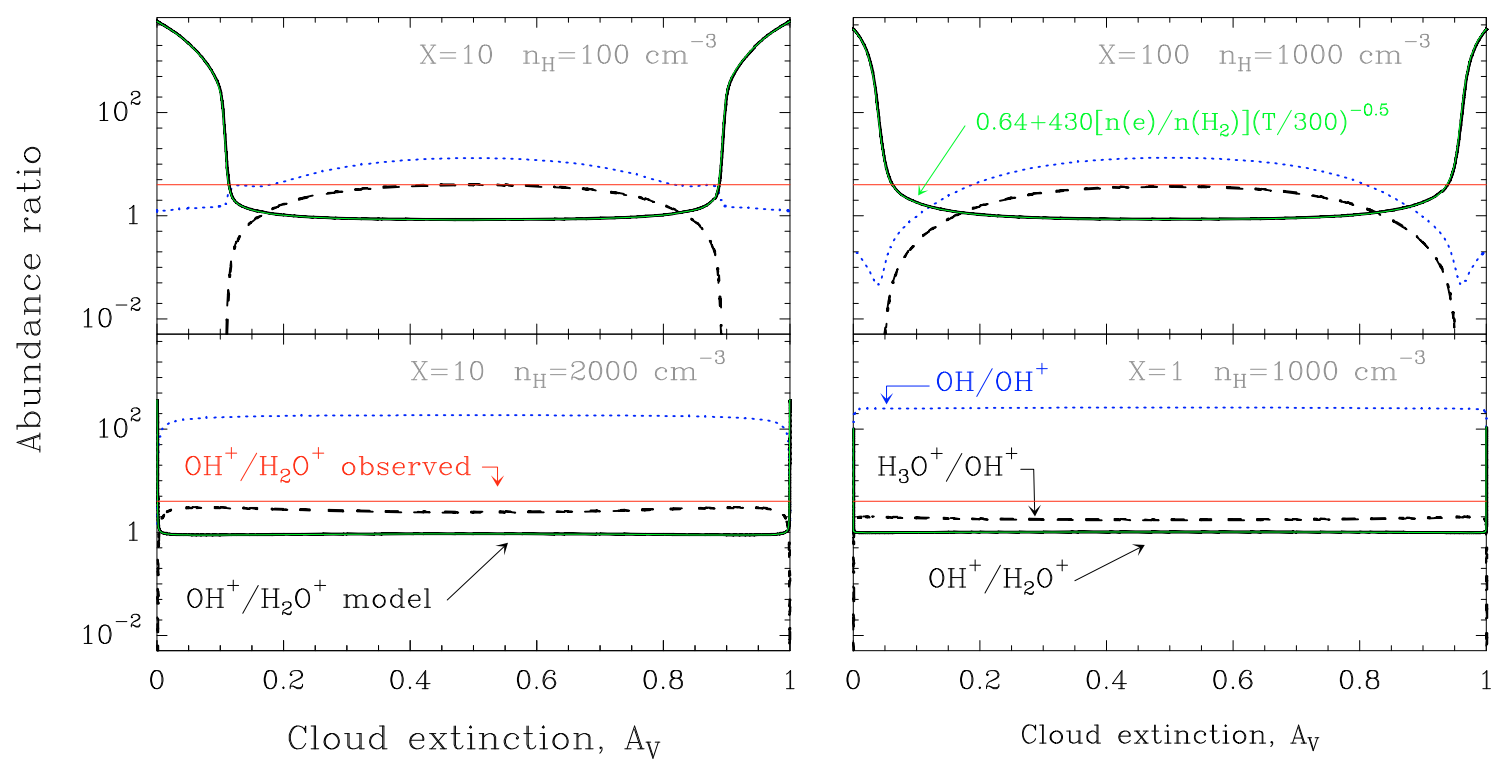

Fig. 2. Predictions of the Meudon-PDR model for a slab of $A_{v}=1$ mag illuminated from both sides. The radiation field and gas density are given in each panel. Full lines: $n\left(\mathrm{OH}^{+}\right) / n\left(\mathrm{H}_{2} \mathrm{O}^{+}\right)$, dotted lines: $n(\mathrm{OH}) / n\left(\mathrm{OH}^{+}\right)$, dashed lines: $n\left(\mathrm{H}_{3} \mathrm{O}^{+}\right) / n\left(\mathrm{OH}^{+}\right)$. Equation (2) determined for each Av in the model is shown in green.

\footnotetext{
1 LERMA, CNRS, Observatoire de Paris and ENS, France e-mail: maryvonne.gerin@ens. fr

2 Chalmers University of Technology, Göteborg, Sweden

3 Centro de Astrobiología, CSIC-INTA, Madrid, Spain

${ }_{5}^{4}$ Depts. of Physics, Astronomy \& Chemistry, Ohio State Univ., USA

5 The Johns Hopkins University, Baltimore, MD 21218, USA

6 JPL, California Institute of Technology, Pasadena, USA

7 California Institute of Technology, Pasadena, CA 91125, USA

${ }^{8}$ Institut d'Astrophysique Spatiale (IAS), Orsay, France

9 I. Physikalisches Institut, University of Cologne, Germany

${ }_{10}$ Laboratoire d'Astrophysique de Marseille (LAM), France

11 Laboratoire d'Astrophysique de Grenoble, France

12 Université de Toulouse; UPS; CESR; 9 avenue du colonel Roche, 31028 Toulouse Cedex 4, France

13 CNRS; UMR5187; 31028 Toulouse, France
}

14 Inst. of Physical Chemistry, Polish Academy of Sciences, Poland

15 Nicolaus Copernicus University, Torún, Poland

16 Tata Institute of Fundamental Research, Mumbai, India

17 Dept. of Physics \& Astronomy, University of Calgary, Canada

18 European Space Astronomy Centre, ESA, Madrid, Spain

19 MPI für Extraterrestrische Physik, Garching, Germany

${ }^{20}$ MPI für Radioastronomie, Bonn, Germany

${ }^{21}$ Gemini telescope, Hilo, Hawaii, USA

22 Nicolaus Copernicus Astronomical Center, Poland

23 SRON Netherlands Institute for Space Research, The Netherlands

24 Sterrewacht Leiden, The Netherlands

25 Deutsches Zentrum für Luft- und Raumfahrt e. V., RaumfahrtAgentur, Bonn, Germany

26 Department of Physics and Astronomy, University of Waterloo, Canada

27 Osservatorio Astrofisico di Arcetri-INAF- Florence, Italy 